\title{
Comparison of Models for Olfactometer Data
}

\author{
A.C. DAVISON and I. RICARD \\ Olfactometer experiments are used to study the responses of arthropods to potential \\ attractants, for purposes such as understanding natural defenses of plants against their \\ herbivores. Such experiments typically lead to multivariate data consisting of small cor- \\ related counts, which are overdispersed relative to standard models. In this paper mod- \\ els that account for the overdispersion under different hypotheses on insect behavior are \\ described and illustrated with an example, and a graphical approach to discriminating \\ among them is briefly discussed. \\ Supplementary files giving technical computations, data and code are available on- \\ line.
}

Key Words: Discrete choice; Kaplan-Meier estimate; Markov process; Overdispersion; Survivor function; Wasp.

\section{INTRODUCTION}

This paper was stimulated by collaboration with colleagues in the Laboratory of Evolutionary Entomology at the University of Neuchâtel, who study interactions between plants, their predators and the predators of the predators. For example, a plant under attack from a caterpillar may release volatiles that attract parasitoid wasps, which then lay their eggs inside the caterpillar, thus reducing the damage to the plant when wasp larvae subsequently hatch and attack the caterpillar from within (Turlings et al. 1995). In other experiments maize roots have been shown to emit volatiles that attract entomopathogenic nematodes, when attacked by insects (Rasmann et al. 2005). Such natural defense mechanisms have the potential both to provide novel strategies for pest management and to aid in understanding the evolution of multitrophic level interactions. This domain of chemical ecology is reviewed by D'Alessandro and Turlings (2006).

The reactions of arthropods to stimuli play a key role in this area, and may be studied in various ways. One important approach is through olfactometer experiments, which we

\footnotetext{
A.C. Davison $(\varangle)$ is Professor, Institute of Mathematics, Station 8, Ecole Polytechnique Fédérale de Lausanne, 1015 Lausanne, Switzerland (E-mail: anthony.davison@epfl.ch). I. Ricard is Statistician, Clinical Trial Unit, University Hospital Basel, Schanzenstrasse 55, 4031 Basel, Switzerland (E-mail: ricard.ingrid@ gmx.fr)
} 
here describe in relation to experiments on parasitoid wasps conducted by our colleagues. An olfactometer consists of a central chamber connected by arms to a number of outside chambers. The arms are constructed so that movement from them back into the central chamber is impossible. The odors of plants, possibly with different treatments applied, are introduced into the outer chambers and wafted along the arms towards the central chamber, into which several wasps are placed. If attracted by the odors in the arms, the wasps may choose to leave the central chamber, tracking an odor into the corresponding arm. After a fixed time the number of wasps that have moved into each arm is counted; we call this a bioassay. Several bioassays can be conducted each day, and after a number of days, over which the treatment configuration is varied according to a systematic experimental design, the counts may be used to compare the attractiveness of different plant-treatment combinations (Turlings, Davison, and Tamò 2004; Tamò et al. 2006). Such apparatus is quite widely used - the Web of Science lists around 1100 papers in entomology, ecology and related fields with 'olfactometer' appearing in the key words-but only Ricard and Davison (2007) seem to have discussed such experiments in the statistical literature.

Consider for example Table 1, which summarizes data collected by Michael Rostas of the University of Neuchâtel on the relative attractiveness to naive wasps of three different sorts of plant treatment, 'herbivore' (h), 'hormone' (b), and both (hb). The full dataset, with $\mathrm{R}$ code for the analysis, is available with the supplementary material. For a given experiment, six plants were used: three treated plants (one for each treatment) and three control plants; the three olfactometer arms with the treatments were alternated with control arms (no). For each bioassay the wasps were released in groups of six and then exposed to the six odors (h, b, hb, no no, no) simultaneously for a period of $T=0.5$ hours. The orientation of the treated arms was changed after every experiment; for technical reasons just one experiment, comprising several bioassays, was performed each day. Each experiment/odor situation was replicated in five bioassays, except for the first which had only four, and eight experiments were performed in total. The data show a strong effect of treatment hb, a smaller effect of $h$, and little or none of $b$. The counts are small but are rather expensive to obtain - the full table represents around three weeks' solid experimentation—so detailed modelling in order to extract the maximum information from them seems justified.

The data are typically overdispersed relative to the simplest, multinomial, model. Quasilikelihood (McCullagh and Nelder 1989, Chapter 9) provides a generic approach to comparing the treatments, but in view of the experimental effort involved we have taken a different route. Ricard and Davison (2007) discuss the design and statistical analysis of olfactometer data based on a Markov chain model that accounts for the possibility that the overdispersion is caused by wasps following each other. One important issue is that collection of data at the end of each bioassay means that the order in which choices are made is lost, thereby reducing the statistical information available and making it hard to compare different hypotheses about wasp behavior. One purpose of the present paper is to investigate whether more complete data would allow such hypotheses to be compared in realistic experiments.

Although the ideas in the paper were developed in a very specific context, discrete choice data arise commonly in economics (Train 2009) as applied in domains such as trans- 
Table 1. Summary data from Michael Rostas' experiment, with the final six columns showing the allocation of odor sources to arms and the corresponding numbers of wasps. The treatments are 'herbivore' (h), 'hormone' (b), 'herbivore+hormone' (hb), and none (no).

\begin{tabular}{|c|c|c|c|c|c|c|c|}
\hline \multirow[b]{2}{*}{ Experiment } & \multirow[b]{2}{*}{ Bioassay } & \multicolumn{6}{|c|}{ Treatment } \\
\hline & & $\mathrm{h}$ & no & $\mathrm{hb}$ & no & $\mathrm{b}$ & no \\
\hline \multirow[t]{5}{*}{1} & & Arm 1 & Arm 2 & Arm 3 & Arm 4 & Arm 5 & Arm 6 \\
\hline & 1 & 0 & 0 & 6 & 0 & 0 & 0 \\
\hline & 2 & 0 & 0 & 5 & 0 & 0 & 1 \\
\hline & 3 & 0 & 0 & 5 & 0 & 0 & 1 \\
\hline & 4 & 0 & 0 & 5 & 0 & 0 & 0 \\
\hline \multirow[t]{6}{*}{2} & & Arm 1 & Arm 2 & Arm 5 & Arm 4 & Arm 3 & Arm 6 \\
\hline & 1 & 0 & 0 & 5 & 0 & 0 & 0 \\
\hline & 2 & 3 & 1 & 2 & 0 & 0 & 0 \\
\hline & 3 & 0 & 0 & 4 & 2 & 0 & 0 \\
\hline & 4 & 3 & 0 & 2 & 0 & 0 & 0 \\
\hline & 5 & 2 & 0 & 2 & 1 & 0 & 0 \\
\hline \multirow[t]{4}{*}{3} & & Arm 6 & Arm 5 & Arm 4 & Arm 3 & Arm 2 & Arm 1 \\
\hline & 1 & 1 & 0 & 5 & 0 & 0 & 0 \\
\hline & 2 & 2 & 0 & 2 & 0 & 1 & 0 \\
\hline & $\vdots$ & $\vdots$ & $\vdots$ & $\vdots$ & $\vdots$ & $\vdots$ & $\vdots$ \\
\hline \multirow[t]{3}{*}{8} & & Arm 6 & Arm 1 & Arm 2 & Arm 3 & Arm 4 & Arm 5 \\
\hline & $\vdots$ & $\vdots$ & $\vdots$ & : & : & : & $\vdots$ \\
\hline & 5 & 0 & 0 & 4 & 0 & 0 & 1 \\
\hline
\end{tabular}

portation (Ben Akiva, Lerman, and Akiva 1985; Garrow 2010) and health (Ryan, Gerard, and Amaya-Amaya 2008), so the work may be of wider interest.

In Section 2 we first describe generic models for olfactometer data and then describe two inhomogeneous Markov chain models, one previously discussed by Ricard and Davison (2007) and one new. Section 3 describes their application to the data in Table 1. In Section 4 we briefly assess the use of graphical methods for distinguishing among the various models, and in Section 5 give a brief conclusion.

\section{MARKOV MODELS}

\subsection{General}

We suppose that there are initially $m$ individuals in state 0 , which corresponds to the central chamber of an olfactometer, and that over the time period $(0, T]$ they may move to states $1, \ldots, p$, which correspond to the arms of the apparatus. The initial rates at which they do so, $\lambda_{1}, \ldots, \lambda_{p}>0$, depend on the attractiveness of the treatments. If the individuals act independently according to a homogeneous Markov process in continuous time (Cox and Miller 1965, Chapter 4), then the time to the choice made by a wasp will be exponential with rate the combined attractivity of the arms, $\Lambda=\lambda_{1}+\cdots+\lambda_{p}$, and the probability that arm $r$ is chosen is $\lambda_{r} / \Lambda$. One can think of there being $p$ independent competing risks for choosing the arms, with exponential distributions with rates $\lambda_{1}, \ldots, \lambda_{p}$; the choice actually made corresponds to the minimum of these independent exponential variables. Since 
under this model the wasps make their choices independently, after a time $T$ the numbers $n_{0}, n_{1}, \ldots, n_{p}$ in the central chamber and arms will follow a multinomial distribution with denominator $m$ and probabilities

$$
\begin{aligned}
& \pi_{0}=\exp (-T \Lambda) \\
& \pi_{1}=\lambda_{1}\{1-\exp (-T \Lambda)\} / \Lambda, \quad \ldots, \quad \pi_{p}=\lambda_{p}\{1-\exp (-T \Lambda)\} / \Lambda .
\end{aligned}
$$

It is straightforward to construct and maximize a likelihood based on (2.1), and hence to perform inferences on the attractivities $\lambda_{r}$. Statistical analysis only of the data for individuals that have made a choice involves using a multinomial distribution with denominator $n=\sum_{r=1}^{p} n_{r}$

$$
n_{1}, \ldots, n_{p} \sim \operatorname{Mult}\left(n ; \lambda_{1} / \Lambda, \ldots, \lambda_{p} / \Lambda\right)
$$

this is the multinomial distribution for $n_{1}, \ldots, n_{p}$ derived from that for $n_{0}, n_{1}, \ldots, n_{p}$. This analysis is very simple but can be appreciably less efficient than use of all the data if $n_{0}$ is sizeable; moreover it allows inference only about the ratios of the $\lambda_{r}$, and not about their values. The information in the counts $n_{0}, n_{1}, \ldots, n_{p}$ depends on $T \Lambda$ : if this is small, then few choices will typically be made and the data will be uninformative about the treatments.

This simple model is often inappropriate because the counts are overdispersed relative to the multinomial distribution. Such overdispersion might be due to general variation of experimental conditions, leading to fluctuations in the parameters $\lambda_{r}$, or to dependence among individuals stemming from their behavior. For example, they may tend to follow each other into the arms of the apparatus, influenced by either visual cues or olfactory cues. In the next subsections we discuss some models that accommodate these possibilities.

\subsection{Dirichlet-Multinomial Model}

One way to introduce overdispersion is to extend the model (2.2) for the numbers of individuals making choices to the Dirichlet-multinomial distribution (Mosimann 1962)

$$
\begin{gathered}
\left(\begin{array}{c}
n \\
n_{1} \ldots n_{p}
\end{array}\right) \frac{\prod_{r=1}^{p} \Gamma\left(\lambda_{r} / \omega+n_{r}\right)}{\prod_{s=1}^{p} \Gamma\left(\lambda_{s} / \omega\right)} \frac{\Gamma(\Lambda / \omega)}{\Gamma(\Lambda / \omega+n)}, \\
n_{1}, \ldots, n_{p} \in\{0,1, \ldots, n\}, \quad \sum n_{r}=n,
\end{gathered}
$$

which may be constructed by (i) supposing that conditional on the probabilities $\pi_{1}, \ldots, \pi_{p}$, the counts $n_{1}, \ldots, n_{p}$ have a multinomial distribution with probabilities $\pi_{1}, \ldots, \pi_{p}$; (ii) supposing that the joint distribution of $\pi_{1}, \ldots, \pi_{p}$ is Dirichlet with parameters $\lambda_{1} / \omega, \ldots, \lambda_{p} / \omega$, where $\omega>0$; and (iii) integrating over the $\pi_{r}$. In this case

$$
\mathrm{E}\left(N_{r}\right)=n \lambda_{r} / \Lambda, \quad \operatorname{var}\left(N_{r}\right)=n \frac{\lambda_{r}\left(\Lambda-\lambda_{r}\right)}{\Lambda^{2}}\left\{1+(n-1) \frac{\omega}{\Lambda+\omega}\right\},
$$

so while the means of the counts equal those under the multinomial model, the variances are increased by an amount that depends on the concentration parameter $\omega$; the multinomial model (2.2) is recovered when $\omega \rightarrow 0$. Expression (2.3) might be held to represent generalized overdispersion corresponding to changes in experimental conditions from one 
bioassay to another, the idea being that for each bioassay the wasps act independently of each other, but variation in the conditions between bioassays induces overdispersion. This standard model provides a baseline against which to compare models constructed specifically for the application.

To simulate data from (2.3), we first generate the number of individuals making a choice, $n$, from a distribution with support on the set $\{0, \ldots, m\}$. Typically the mass function for this distribution will depend on $T$; below we use the distribution for the total number of choices for the model in Section 2.3. We then generate probabilities from the Dirichlet distribution, then generate the numbers of individuals $n_{1}, \ldots, n_{p}$ choosing the different arms using the multinomial distribution with denominator $n$ and the simulated probabilities, and finally generate the times of the choices using the property that in a homogeneous Poisson process on the interval $(0, T)$ in which $n_{r}$ events are known to occur, the event times are independently distributed according to the $U(0, T)$ distribution.

\subsection{Olfactory Cues}

In a second approach to accounting for the overdispersion, Ricard and Davison (2007) suggest that dependence between the successive choices made by wasps may be induced as follows: the presence of one or more wasps in an arm of the olfactometer may increase its attractiveness for individuals still in the central chamber. Their mathematical formulation of this changes the simple homogeneous Markov process model in Section 2.1 into an inhomogeneous one, in which the rate parameter for a chosen arm increases after each choice. If $\lambda_{r}$ is the rate parameter for the $r$ th arm in the absence of wasps, the model postulates that this is increased to $\lambda_{r}+a \omega$ when $a$ wasps have already chosen the arm, and the parameter $\omega \geq 0$ represents the attraction due to a single wasp. For example, suppose that $p=3$ and three choices are made successively, of arm 1, arm 1, and arm 2. Initially the attractivities of the arms are $\lambda_{1}, \lambda_{2}, \lambda_{3}$, changing after the first choice to $\lambda_{1}+\omega, \lambda_{2}, \lambda_{3}$, after the second choice to $\lambda_{1}+2 \omega, \lambda_{2}, \lambda_{3}$, and after the third choice to $\lambda_{1}+2 \omega, \lambda_{2}+\omega, \lambda_{3}$. The motivation for such a model is that olfactory cues, due for example to pheromones, might strengthen the attractiveness of arms that have already been chosen, and that this attraction might increase with the number of previous choices of the arm.

The simplest way to derive the joint distribution of the counts $N_{1}, \ldots, N_{p}$ in the arms at time $T$ is to include the times of the successive choices $S_{1}, \ldots, S_{n}$, and then to integrate them out. Under this model the probability element associated to the event $N_{1}=n_{1}, \ldots, N_{p}=n_{p}, S_{1}=s_{1}, \ldots, S_{n}=s_{n}$, with $m-n$ individuals remaining, is

$$
\frac{m !}{(m-n) !}\left(\begin{array}{c}
n \\
n_{1} \ldots n_{p}
\end{array}\right) \times \prod_{r=1}^{p}\left\{\prod_{j=0}^{n_{r}-1}\left(\lambda_{r}+j \omega\right)\right\}^{\delta_{0}\left(n_{r}\right)} \times \prod_{k=0}^{n} e^{-(m-k)(\Lambda+k \omega)\left(s_{k+1}-s_{k}\right)}
$$

where $\delta_{0}(j)=1$ if $j>0$ and equals zero otherwise, $s_{0}=0$ and $s_{n+1}=T$. A derivation of this expression is given in Ricard and Davison (2007). Unfortunately it cannot be used for inference, because the times of choices are typically unavailable, so inference must be based on $N_{1}, \ldots, N_{p}$ only. On integrating (2.4) over the set $0<s_{1}<\cdots<s_{n}<T$ we find 
that the probability mass function $\operatorname{Pr}\left(N_{1}=n_{1}, \ldots, N_{p}=n_{p}\right)$ for the counts is

$$
\begin{aligned}
& \left(\begin{array}{c}
m \\
n_{1} \ldots n_{p}
\end{array}\right) \prod_{r=1}^{p}\left\{\prod_{k=0}^{n_{r}-1}\left(\lambda_{r}+k \omega\right)\right\}^{\delta_{0}\left(n_{r}\right)} e^{-(\Lambda+n \omega)(m-n) T} \\
& \times \sum_{i=0}^{n} \frac{\left(\begin{array}{c}
n \\
i
\end{array}\right)(-1)^{i} e^{-i\{\Lambda-\omega(m-2 n+i)\} T}}{\prod_{j=0, j \neq n-i}^{n}\{\Lambda-\omega(m+i-n-j)\}}
\end{aligned}
$$

The conditional mass function for those individuals that change state may be shown to be

$$
\begin{aligned}
& \operatorname{Pr}\left(N_{1}=n_{1}, \ldots, N_{p}=n_{p} \mid \sum N_{r}=n\right) \\
& \quad=\left(\begin{array}{c}
n \\
n_{1} \ldots n_{p}
\end{array}\right) \frac{\prod_{r=1}^{p}\left\{\prod_{i=0}^{n_{r}-1}\left(\lambda_{r}+i \omega\right)\right\}^{\delta_{0}\left(n_{r}\right)}}{\left\{\prod_{i=0}^{n-1}(\Lambda+i \omega)\right\}^{\delta_{0}(n)}},
\end{aligned}
$$

which reduces to (2.3) after a little algebra; this implies that the full likelihood (2.5) rather than the conditional likelihood (2.6) must be used if the Dirichlet-multinomial model for generic overdispersion is to be distinguished from the model for olfactory cues discussed in the present section. If appropriate data are available, then likelihood inference is readily performed based on (2.4), (2.5), or (2.6).

The model described in this section allows a detailed comparison of the information in different observation schemes for olfactometer experiments. The first and most complete scheme requires continuous-time observation of the apparatus, and records both choices and the times at which they were made, corresponding to (2.4), but is demanding for the biologists and thus is not currently performed. A second scheme that uses the data $n_{0}, n_{1}, \ldots, n_{p}$ after a fixed time $T$, corresponding to (2.5), is preferred because it does not involve continuous monitoring and provides a good compromise between experimental and statistical efficiency. A third approach based only on the data $n_{1}, \ldots, n_{p}$, corresponding to the density (2.6), was used by the experimenters before statisticians became involved, but can involve appreciable loss of information relative to (2.5). Further details may be found in Ricard and Davison (2007) and Ricard (2008).

It is straightforward to simulate data from this model using its definition and standard simulation techniques for continuous-time Markov chains (Ripley 1987, p. 104), noting that the rate corresponding to an arm is updated when it is chosen, and that the algorithm is terminated at time $T$.

\subsection{Visual Cues}

A different way to model the idea that wasps may follow each other is to suppose that the only influence on a choice is the preceding choice, due for example to visual cues or to transitory chemical traces. In this case the rate parameters of the inhomogeneous Markov chain at a given time might be $\lambda_{r}+\omega$ for the arm most recently chosen and $\lambda_{s}$ for all other arms. The parameter $\omega$ again represents the attraction of a single wasp but this new model yields a different likelihood from those above. 
To sketch how the likelihood is constructed, suppose that $n \in\{1, \ldots, m\}$ and let $0<$ $s_{1}<\cdots<s_{n}<T$ denote the ordered times at which individuals make their choices. Suppose that the $(j+1)$ st individual opts for state $r$ at time $s_{j+1}$. From time $s_{j}$ onwards, the waiting times in the central chamber before choosing one of the different states $1, \ldots, p$ are independent exponential variables with parameters $\lambda_{1}+\delta_{1}^{j} \omega, \ldots, \lambda_{p}+\delta_{p}^{j} \omega$ respectively, where $\delta_{r}^{j}$ equals one if the $j$ th individual chose the $r$ th state and equals zero otherwise. The waiting time to the first choice is exponential with rate $\Lambda$, and the times between subsequent choices are exponential with rate $\Lambda+\omega$. As the $m-j$ individuals yet to make a choice act independently, the density contribution associated with the interval $\left(s_{j}, s_{j+1}\right]$ between the $j$ th and $(j+1)$ th choices is

$$
\begin{cases}\left(\lambda_{r}+\delta_{r}^{j} w\right) e^{-(\Lambda+w)\left(s_{j+1}-s_{j}\right)(m-j)}, & j \neq 0, \\ \lambda_{r} e^{-m \Lambda s_{1}}, & j=0 .\end{cases}
$$

To understand the form of the probability element associated to the event $N_{1}=n_{1}, \ldots$, $N_{p}=n_{p}, S_{1}=s_{1}, \ldots, S_{n}=s_{n}$, consider a simple example in which $n=3, n_{1}=2$ and $n_{2}=1$. Then the possible orders for the three choices are $(1,1,2),(1,2,1)$ and $(2,1,1)$, and so the probability element is proportional to the product of

$$
\lambda_{1}\left(\lambda_{1}+\omega\right) \lambda_{2}+\lambda_{1} \lambda_{2} \lambda_{1}+\lambda_{2} \lambda_{1}\left(\lambda_{1}+\omega\right)
$$

with

$$
e^{-m \Lambda s_{1}} \times e^{-(m-1)(\Lambda+w)\left(s_{2}-s_{1}\right)} \times e^{-(m-2)(\Lambda+w)\left(s_{3}-s_{2}\right)} \times e^{-(m-3)(\Lambda+w)\left(T-s_{3}\right)} .
$$

The three terms in (2.8) correspond to the $3 ! /(2 ! 1 !)=3$ possible orders for the choices, and the terms in (2.9) represent the contributions of the successive waiting times between the three choices and then from the last choice to the end of the bioassay.

Further thought shows that the general form of the likelihood is

$$
\begin{aligned}
& \frac{m !}{(m-n) !}\left[\left\{\sum_{i=1}^{\left(\begin{array}{c}
n \\
n_{1} \cdots n_{p}
\end{array}\right)} g\left(C_{\left(n_{1}, \ldots, n_{p}\right)}^{i}, \omega, \lambda\right)\right\} e^{-m \Lambda s_{1}}\right. \\
& \left.\times \prod_{k=1}^{n} e^{-(m-k)(\Lambda+\omega)\left(s_{k+1}-s_{k}\right)}\right]^{\delta_{0}(n)}\left(e^{-\Lambda T}\right)^{1-\delta_{0}(n)},
\end{aligned}
$$

where $s_{0}=0, s_{n+1}=T$ and the function $g$ accounts for sums such as (2.8). The quantity $C_{\left(n_{1}, \ldots, n_{p}\right)}^{i}$ represents the $i$ th possible ordering for the choices leading to totals $n_{1}, \ldots, n_{p}$ in the arms, for $i=1, \ldots,\left(\begin{array}{c}n \\ n_{1} \cdots n_{p}\end{array}\right)$, where $\left(\begin{array}{c}n \\ n_{1} \cdots n_{p}\end{array}\right)=n ! /\left(n_{1} ! n_{2} ! \cdots n_{p} !\right)$. For instance, $C_{2,1}^{1}=(1,1,2), C_{2,1}^{2}=(1,2,1)$ and $C_{2,1}^{3}=(2,1,1)$ in the example above, and $g\left(C_{2,1}^{1}, \omega, \lambda\right)=\lambda_{1}\left(\lambda_{1}+\omega\right) \lambda_{2}, g\left(C_{2,1}^{2}, \omega, \lambda\right)=\lambda_{1} \lambda_{2} \lambda_{1}$, and $g\left(C_{2,1}^{3}, \omega, \lambda\right)=\lambda_{2} \lambda_{1}\left(\lambda_{1}+\omega\right)$. The computation of (2.10) is a little involved (Ricard 2008), but the principle should be clear.

A simpler observation scheme records only the final numbers $n_{0}, n_{1}, \ldots, n_{p}$ in the chambers. The corresponding probability mass function can be obtained by integrating 
(2.10) over $s_{1}, \ldots, s_{n}$, yielding

$$
\begin{aligned}
\operatorname{Pr}\left(N_{1}=\right. & \left.n_{1}, \ldots, N_{p}=n_{p}\right) \\
= & \frac{m !}{(m-n) !}\left(e^{-m \Lambda T}\right)^{\delta_{0}(n)}\left\{e^{-(m-n)(\Lambda+\omega) T}\right\}^{1-\delta_{0}(n)} \\
& \times\left[\frac{\sum_{i=1}^{\left(n_{1}^{n} \ldots n_{p}\right)} g\left\{C_{\left(n_{1} \ldots, n_{p}\right)}^{i}, \omega, \lambda\right\}}{(\Lambda+\omega)^{n-1}}\right. \\
& \left.\times\left\{\sum_{i=0}^{n-1} \frac{(-1)^{n-i}}{i !(n-1-i) !} \frac{e^{-T\{n \Lambda+(n-m) \omega\}}-e^{(i-n+1) T(\Lambda+\omega)}}{(i+1) \Lambda-(m-1-i) \omega}\right\}\right]^{1-\delta_{0}(n)},
\end{aligned}
$$

which is analogous to (2.5). When this expression is conditioned on the total number of individuals that changed states, we do not obtain the Dirichlet-multinomial density (2.3), but

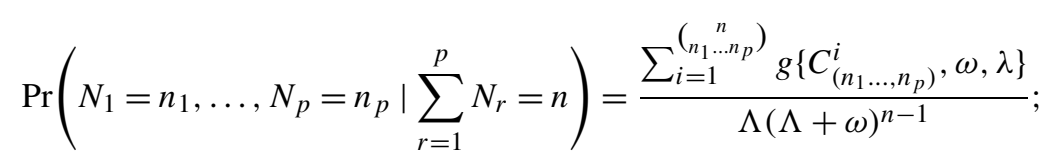

this reduces to the multinomial distribution when $\omega=0$.

Details of the calculations leading to formulae (2.11) and (2.12) may be found in the supplementary material. Simulations for this model may be performed using the principles of Section 2.3.

\section{DATA ANALYSIS}

A simple model for the data in Table 1 takes the attractiveness of the treatment in an arm labelled $r$ to be

$$
\lambda_{r}=\exp \left(\mu+\beta_{r}\right), \quad r \in\{\mathrm{no}, \mathrm{h}, \mathrm{b}, \mathrm{hb}\}, \quad \beta_{\mathrm{no}}=0,
$$

so that $\mu$ represents the attractiveness of an empty arm, and treatment effects such as $\beta_{\mathrm{h}}$ are measured relative to this. The results from likelihood fits of various models to the data are given in Table 2, with standard errors obtained from the inverse observed information matrix, obtained by numerical differentiation (Davison 2003, Chapter 4). The first two models do not allow for overdispersion, the quasi-Poisson fit makes a generic allowance for overdispersion and is included only for comparison, and the final four models are based on the discussion in Sections 2.3, 2.4.

The multinomial model and mass functions (2.3) and (2.12) are fitted to the data only for those wasps that leave the central chamber; the latter two models have almost identical maximized log likelihoods but the log likelihood differences of around 10 indicates that they fit much better than the multinomial model, owing to their capacity to model the overdispersion. When overdispersion is taken into account the parameter estimates barely change but the standard errors increase substantially, though the overall conclusions that treatment $\mathrm{hb}$ is much more attractive than is treatment $\mathrm{h}$, with treatment $\mathrm{b}$ coming a poor 
Table 2. Fits to the data in Table 1 of model (3.1) using different response distributions. Columns $2-6$ show the parameter estimates (standard errors), column 7 gives the maximized log likelihood, and columns 8 and 9 contain the number of parameters $d$ and the AIC value if available. The models are: a quasi-Poisson fit to all the data using the $\mathrm{R}$ function $\mathrm{g} \mathrm{Im}$ with quasi-Poisson variance function; a multinomial fit only to those wasps making choices; fits of (2.3) and (2.12) to those wasps making choices; and fits of (2.1), (2.5) and (2.11) to all the data. The parameter $\mu$ is not estimable from fits only to those wasps making choices.

\begin{tabular}{|c|c|c|c|c|c|c|c|c|}
\hline Model & $\mu$ & $\beta_{\mathrm{b}}$ & $\beta_{\mathrm{h}}$ & $\beta_{\mathrm{hb}}$ & $\log \omega$ & $\hat{\ell}$ & $d$ & AIC \\
\hline quasi-Poisson & 0 (imposed) & $-0.85(0.65)$ & $1.02(0.33)$ & $2.69(0.25)$ & - & - & 3 & - \\
\hline Multinomial & 0 (imposed) & $-0.85(0.53)$ & $1.02(0.27)$ & $2.69(0.21)$ & - & -134.92 & 3 & 275.8 \\
\hline$(2.3)$ & 0 (imposed) & $-0.88(0.62)$ & $0.84(0.34)$ & $2.62(0.25)$ & $1.32(0.40)$ & -124.854 & 4 & 257.7 \\
\hline$(2.12)$ & 0 (imposed) & $-0.88(0.62)$ & $0.84(0.34)$ & $2.62(0.25)$ & $2.21(0.39)$ & -124.846 & 4 & 257.7 \\
\hline$(2.1)$ & $-1.75(0.19)$ & $-0.85(0.53)$ & $1.02(0.27)$ & $2.69(0.21)$ & $\omega=0$ (imposed) & -184.48 & 4 & 377.0 \\
\hline$(2.5)$ & $-2.04(0.22)$ & $-0.89(0.62)$ & $0.86(0.34)$ & $2.64(0.25)$ & $-0.82(0.29)$ & -174.62 & 5 & 354.2 \\
\hline$(2.11)$ & $-2.01(0.22)$ & $-0.88(0.62)$ & $0.84(0.34)$ & $2.62(0.25)$ & $0.20(0.29)$ & -174.30 & 5 & 353.6 \\
\hline
\end{tabular}

third, remain unaltered. Treatments $\mathrm{hb}$ and $\mathrm{h}$ are significantly more attractive than an empty arm, but $b$ is not.

The mass functions (2.1), (2.5) and (2.11) are fitted to the counts $\left\{n_{0}, \ldots, n_{p}\right\}$. The first of these does not allow for the overdispersion but the others do, and again the log likelihood increases by around 10 when the overdispersion is modelled. The log likelihoods show that these data do not allow us to distinguish between the Markov model in Section 2.3 in which the behavior of the wasps depends on all previous choices, and that in Section 2.4 in which it depends only on the single preceding choice: this second model is very marginally favored, with a log likelihood around 0.01 higher for the choice-only data and around 0.3 higher for all the data, but no firm conclusion could be drawn from these differences. The chief difference between the estimated parameters for the models is in the value of $\hat{\omega}$, which is appreciably larger for the visual cue model, presumably in order to generate roughly the same levels of overdispersion as in the olfactory cue model.

Table 2 contains the AIC values for the models, but they are of little use in this context, because not all the fits are based on the same data. Leaving the quasi-Poisson fit aside because it does not provide a log likelihood, the next three models are based only on the wasps making choices, and the final three are based on all the data. Thus these two groups of models are not directly comparable, a situation that also arises in the setting of restricted likelihood estimation (Vaida and Blanchard 2005), or, more generally, where conditioning or margining arguments are used to eliminate parts of the overall likelihood. Within the two groups the conclusions based on AIC are the same as those based on the likelihood ratio statistics.

A more informal approach to model assessment would be the use of residuals, but in the present case this is uninformative. As the data consist of small counts, residuals are very discrete, and their power for discriminating between models is low. Thus although it is possible to detect the overdispersion, discrimination between the competing Markov models of Sections 2.2-2.4 based only on the data in Table 1 seems to be impossible. It is therefore natural to ask whether it would be possible to distinguish between these models, 
if data on the choice times had been recorded, for example using a video camera. Would it be worthwhile to record these times, in order to shed further light on the behavior of wasps in the olfactometer? We turn to this in the following section.

\section{GRAPHICAL COMPARISON OF MODELS}

In this section we consider how data on the times of choices, if available, would aid in distinguishing between the three models described in Sections 2.2-2.4, corresponding to general overdispersion, or to overdispersion due to the response of the wasps to olfactory or visual cues. Although there may be other bases for the last two models, it will be convenient to refer to them in these terms. We discuss a graphical approach, as being both exploratory and potentially capable of showing that neither model would be appropriate, though in practice it should be supplemented with the maximized values of the log likelihoods (2.4) for the olfactory model and (2.10) for the visual model.

Under each of these models the inter-event times are independent exponential variables, whose parameters we can estimate. For example, if we have three choice times $0<S_{1}<$ $S_{2}<S_{3}<T=0.5$ (hours) and estimates $\hat{\Lambda}$ and $\hat{\omega}$, then approximately

$$
m \hat{\Lambda} S_{1},(m-1) \hat{\Lambda}\left(S_{2}-S_{1}\right),(m-2) \hat{\Lambda}\left(S_{3}-S_{2}\right) \stackrel{\text { iid }}{\sim} \exp (1)
$$

for data generated according under the Dirichlet-multinomial model of Section 2.2,

$$
m \hat{\Lambda} S_{1},(m-1)(\hat{\Lambda}+\hat{\omega})\left(S_{2}-S_{1}\right),(m-2)(\hat{\Lambda}+2 \hat{\omega})\left(S_{3}-S_{2}\right) \stackrel{\mathrm{iid}}{\sim} \exp (1)
$$

for data generated according under the olfactory cue model of Section 2.3, and

$$
m \hat{\Lambda} S_{1},(m-1)(\hat{\Lambda}+\hat{\omega})\left(S_{2}-S_{1}\right),(m-2)(\hat{\Lambda}+\hat{\omega})\left(S_{3}-S_{2}\right) \stackrel{\mathrm{iid}}{\sim} \exp (1)
$$

for data generated according to the visual cue model of Section 2.4, while the quantities $\left.(m-3) \hat{\Lambda}\left(T-S_{3}\right),(m-3) \hat{(} \Lambda+3 \hat{\omega}\right)\left(T-S_{3}\right)$ and $\left.(m-3) \hat{(} \Lambda+\omega\right)\left(T-S_{3}\right)$ corresponding to the interval $\left(S_{3}, T\right]$ should be treated as censored unit exponential variables. This suggests that a Kaplan-Meier (1958) estimator of the log-survivor function based on the quantities

$$
\left\{(m-j) \hat{\theta}_{j}\left(S_{j+1}^{*}-S_{j}^{*}\right), C_{j+1}\right\}, \quad j=0, \ldots, n,
$$

where $S_{0}=0, S_{j}^{*}=S_{j}$ if $S_{j}$ is observed, $S_{n+1}^{*}=T$ is censored and the indicator variable $C_{j+1}=I\left(S_{j+1} \leq T\right)$ indicates whether $S_{j+1}$ is observed, and $\hat{\theta}_{j}$ depends on the model to be tested, will have slope -1 for a correct model.

To assess the usefulness of such plots we simulated data for experiments with 50 bioassays, corresponding to around ten experiments, comparable with the data in Table 1, with parameter values taken from fits to these data. Figure 1 shows results for data simulated from the Dirichlet-multinomial distribution (2.3), when the model representing olfactory cues is fitted based only on the counts and based on both counts and choice times, i.e. using (2.5) and (2.4). In this case it is possible to detect model inadequacy based on the first two inter-choice times, but the censoring of later times is too heavy for them to be useful. More 

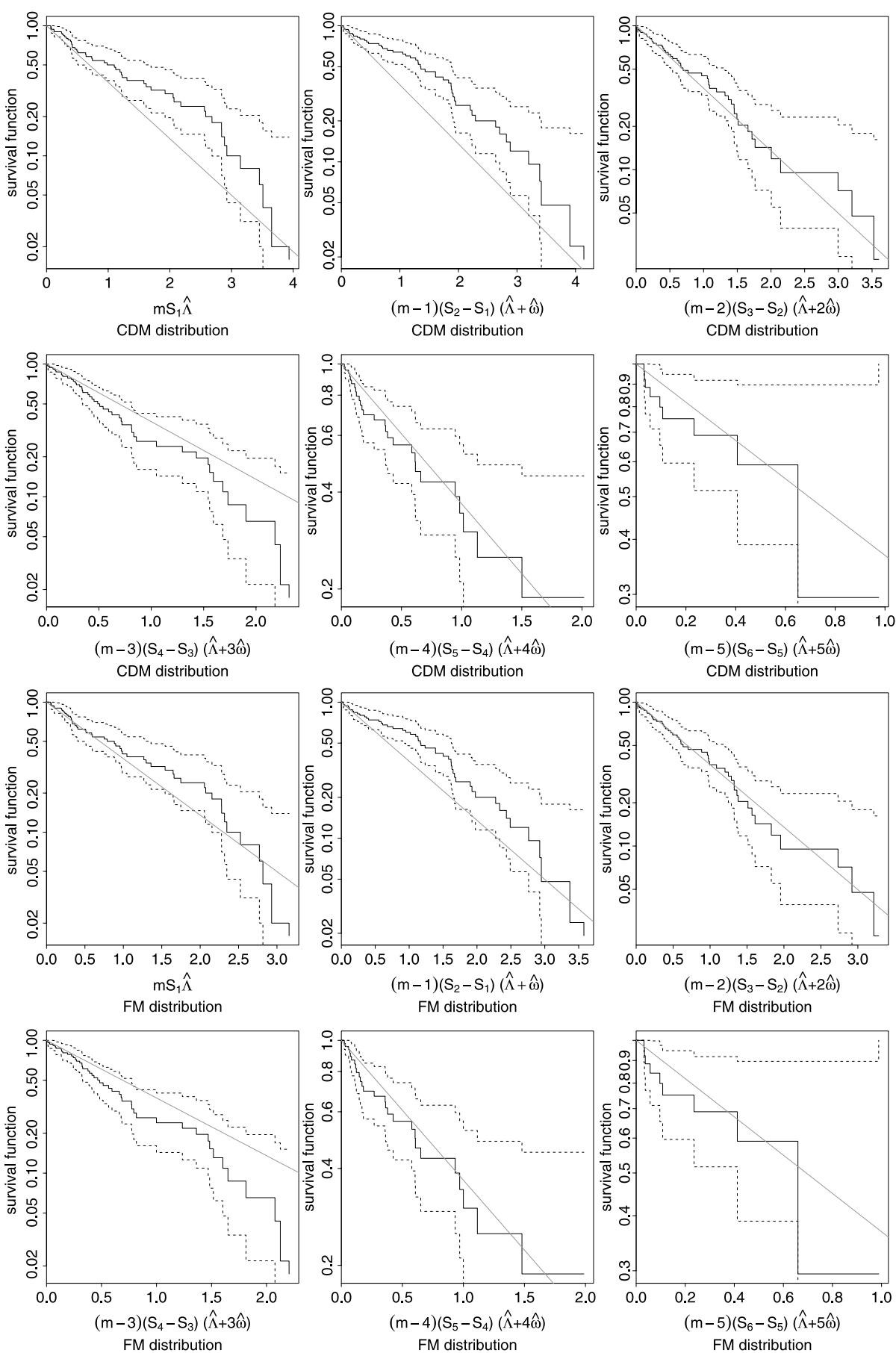

Figure 1. Kaplan-Meier estimates (solid) and 95\% confidence intervals (dashes) for the scaled inter-event times when 50 bioassays are performed. Each set of six panels (from left to right, top to bottom), show plots for $m S_{1} \hat{\Lambda}$, $(m-1)\left(S_{2}-S_{1}\right)(\hat{\Lambda}+\hat{\omega}), \ldots,(m-5)\left(S_{6}-S_{5}\right)(\hat{\Lambda}+5 \hat{\omega})$. The data are generated under the Dirichlet-multinomial model. The gray line represents the theoretical survival function, i.e. $t \mapsto\{\exp (-t)\}$. The parameters were estimated using (2.5) for the six upper panels and using (2.4) for the six lower panels. The $y$ axis is on a log scale. 
extensive simulations and plots for this and other scenarios suggest that it is usually possible to detect differences between the Dirichlet-multinomial and the non-homogeneous models based on such data, though slightly larger datasets are needed for the model of Section 2.4 than for that of Section 2.3.

The question remains whether it would be feasible to use this approach to discriminate the olfactory and visual cue models. The inter-event time that will provide the most information in this case is that for the first and second events $(m-1)\left(S_{2}-S_{1}\right)(\hat{\Lambda}+\hat{\omega})$, for which censoring is generally small. The estimated value of $\omega$ under the visual model is appreciably bigger than under the olfactory model, so it may be possible to use these times to tell the models apart. Unfortunately simulations suggest that this will only rarely be possible, so that a more powerful statistical approach must be sought, or some appeal made to experimental knowledge, if these models are to be distinguished. In practice it seems to be infeasible to perform very large experiments.

\section{DISCUSSION}

We have described two existing classes of models which can account for overdispersion seen in olfactometer data, and introduced a third class. Two of the classes are based on inhomogeneous Markov chains with slightly different assumptions. Olfactometer data show clear overdispersion, and the models make appropriate allowance for this when estimating parameters for effects of interest. Data on the times of successive choices are unavailable to us at present, but simulations suggest that if they were, it would be possible to discriminate between models with general overdispersion due to changes in experimental conditions, and those in which the overdispersion stems from dependence among the choices, based on samples of realistic sizes. It would be appreciably more difficult to discriminate between the different inhomogeneous Markov chain models, however. The availability of data on choice times would also allow model assumptions to be tested.

\section{SUPPLEMENTARY MATERIALS}

The following supplementary materials are available online:

File containing the computation of (2.11). (PDF file)

Data for experiment summarized in Table 1 of the paper. (ASCII file)

$\mathrm{R}$ code needed to produce Table 2 of the paper. (ASCII file)

$\mathrm{R}$ script with code needed to produce Figure 1 of the paper. (R script file)

\section{ACKNOWLEDGEMENTS}

We thank Ted Turlings, two referees, an associate editor and the editor for helpful comments and Michael Rostas for access to his data. The work was supported by the Swiss National Science Foundation through the National Centre for Competence in Research on Plant Survival (http://www2.unine.ch/nccr). 


\section{REFERENCES}

Ben Akiva, M., Lerman, S., and Akiva, B. (1985), Discrete Choice Analysis: Theory and Application to Travel Demand, Cambridge: MIT Press.

Cox, D. R., and Miller, H. D. (1965), The Theory of Stochastic Processes, London: Chapman \& Hall.

D’Alessandro, M., and Turlings, T. C. J. (2006), “Advances and Challenges in the Identification of Volatiles That Mediate Interactions Among Plants and Arthropods," The Analyst, 131, 24-32.

Davison, A. C. (2003), Statistical Models, Cambridge: Cambridge University Press.

Garrow, L. A. (2010), Discrete Choice Modelling and Air Travel Demand: Theory and Applications, Farnham: Ashgate.

Kaplan, E. L., and Meier, P. (1958), "Nonparametric Estimation From Incomplete Observations," Journal of the American Statistical Association, 53, 457-481.

McCullagh, P., and Nelder, J. A. (1989), Generalized Linear Models (2nd ed.), London: Chapman \& Hall.

Mosimann, J. E. (1962), "On the Compound Multinomial Distribution, the Multivariate Beta Distribution, and Correlations Among Proportions," Biometrika, 49, 65-82.

Rasmann, S., Köllner, T. G., Degenhardt, J., Hiltpold, I., Töpfer, S., Kuhlmann, U., Gershenzon, J., and Turlings, T. C. J. (2005), "Recruitment of Entomopathogenic Nematodes by Insect-Damaged Maize Roots," Nature, $434,732-737$.

Ricard, I. (2008), "Statistical Methods for Insect Choice Experiments", Ph.D. thesis, Ecole Polytechnique Fédérale de Lausanne, Switzerland.

Ricard, I., and Davison, A. C. (2007), "Statistical Inference for Olfactometer Data," Applied Statistics, 56, 479492.

Ripley, B. D. (1987), Stochastic Simulation, New York: Wiley.

Ryan, M., Gerard, K., and Amaya-Amaya, M. (eds.) (2008), Using Discrete Choice Experiments to Value Health and Health Care, Berlin: Springer.

Tamò, C., Ricard, I., Held, M., Davison, A. C., and Turlings, T. C. J. (2006), “A Comparison of Naive and Conditioned Responses of Three Generalist Endoparasitoids of Lepidopteran Larvae to Host-Induced Plant Odours," Animal Biology, 56, 205-220.

Train, K. (2009), Discrete Choice Models with Simulation (2nd ed.), Cambridge: Cambridge University Press.

Turlings, T. C. J., Davison, A. C., and Tamò, C. (2004), "A Six-Arm Olfactometer Permitting Simultaneous Observation of Insect Attraction and Odour Trapping," Physiological Entomology, 29, 45-55.

Turlings, T. C. J., Loughrin, J. H., Röse, U., McCall, P. J., Lewis, W. J., and Tumlinson, J. H. (1995), "How Caterpillar-Damaged Plants Protect Themselves by Attracting Parasitic Wasps," Proceedings of the National Academy of Sciences, USA, 92, 4169-4174.

Vaida, F., and Blanchard, S. (2005), "Conditional Akaike Information for Mixed-Effects Models," Biometrika, 92, 351-370. 